\title{
Implementing Interference Cancellation to Increase the EV-DO Rev A Reverse Link Capacity
}

\author{
Jilei Hou, John E. Smee, Henry D. Pfister, and Stefano Tomasin, QUALCOMM Inc.
}

\begin{abstract}
This article provides the principles and practice of how interference cancellation can be implemented on the EV-DO Rev A reverse link. It is shown that applying interference cancellation to CDMA achieves the multiple access channel sum rate capacity for either frame synchronous or asynchronous users. The per user SINR gain from space-time interference cancellation translates directly into a CDMA capacity gain of the same factor, allowing EV-DO Rev A to support more users with higher data rates. We demonstrate how interference cancellation can be added to base station processing without modifying user terminals, EV-DO standards, or network coverage. We present commercially viable receiver architectures for implementing interference cancellation with the asynchronism and H-ARQ of EV-DO RevA, and explain why closed loop power control can operate the same way it does today. Network level simulations over a wide range of channels confirm that interference cancellation offers significant capacity gains for all users, while maintaining the same link budget and system stability.
\end{abstract}

\section{INTRODUCTION}

The EV-DO Revision A system [1] was designed to support applications ranging from wireless internet connectivity to VoIP, video telephony, and broadcast services over a wide area cellular network [2]. On the EV-DO reverse link, codedivision multiple access (CDMA) has been shown to offer high spectral efficiency, low latency, and system robustness [3]. Implemented with universal frequency reuse and power control, CDMA allows each user to transmit across a wide bandwidth, and achieve soft handoff across base stations and softer handoff across multiple sectors of the same cell. Hybrid automatic repeat request (H-ARQ) further improves each user's spectral efficiency in fading channels and facilitates lowlatency transmission for delay-sensitive applications by targeting early packet termination. High spectral efficiency is achieved by efficient per user transmissions combined with a closed loop medium access control (MAC) algorithm that maximizes the received signal level at each base station while maintaining good coverage across the network. This article explains the theory and practice of further increasing CDMA reverse link capacity by adding interference cancellation (IC) to the base station receiver and integrating IC into the system operation.

The multiuser detection research area covers many different algorithms for mitigating interference between users [4]. We consider techniques that reconstruct and subtract decoded signals from the receiver sample buffer. This notion of IC is well known and was proposed for CDMA at least 15 years ago [5]. While classical IC often assumes frame synchronous transmissions, most networks intentionally stagger user data frames uniformly in time. In this article we show that asynchronous IC in theory achieves sum rate capacity. In addition, we demonstrate how IC can be applied to commercial CDMA systems that incorporate H-ARQ (e.g., EV-DO RevA and wideband CDMA [WCDMA] EUL) and how the inherent asynchronous nature of the transmissions simplifies the problem.

From an implementation standpoint, it is simplest to cancel signals whose modulation symbols are a priori known at the base station (e.g., pilot signals) because the IC process can avoid data demodulation and decoding. The most advanced forms of IC with the largest capacity gains require data directed cancellation of all decoded signals. IC is no longer only merely a topic of academic research as technology advancements have made CDMA IC commercially viable. For example, the QUALCOMM CSM6800 chipset solution for the EV-DO RevA reverse link offers pilot IC. Significantly larger gains are possible when decoded packets are reconstructed and removed. Designs for combined pilot, overhead, and traffic IC are being developed for existing standards such as EV-DO.

This article is structured as follows. The next section describes the theoretical motivation and practical aspects of implementing IC, followed by 


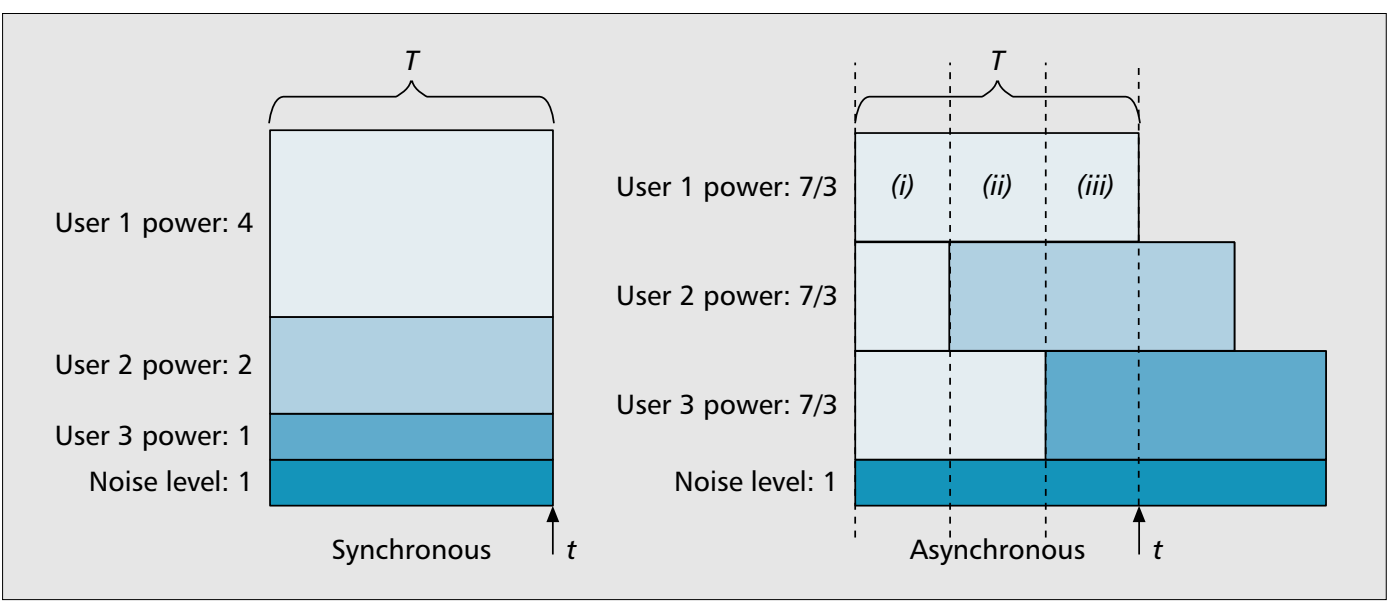

Figure 1. Decoding timelines for frame synchronous and frame asynchronous.

a section on receiver architectures. We then provide a section that outlines the network level simulation modeling of IC and provide capacity results. The final section contains our conclusions.

\section{PRINCIPLES OF INTERFERENCE CANCELLATION}

We begin with a simple example showing how the sum rate capacity can be achieved by successive IC (SIC) for a frame asynchronous CDMA system. We introduce the concept of effective rise-over-thermal (ROT) control for CDMA networks with IC. After discussing the information theory aspects of the CDMA reverse link with multiple receiver antennas and IC, we describe how to integrate IC with the power control, asynchronism, and H-ARQ of EV-DO RevA.

\section{ACHIEVIng Sum Rate CAPaCITY WITH CDMA AND SUCCESSIVE INTERFERENCE CANCELLATION: FrAME SYNCHRONOUS VS. ASYNCHRONOUS}

It is well known that the sum rate capacity can be achieved through SIC for frame synchronous equal rate users with an exponential power profile [5]. However, in practical systems users often transmit asynchronously because the processing power and network bandwidth at the receiver will have a more uniform usage profile in time. In contrast, frame synchronism among users requires a burst of processing power and network resources at the end of each frame boundary since all users finish packets at the same time. In classic SIC with exponential power shaping and uniform data rates, the last user is received with very low power and is overly dependent on the successful decoding of prior users. Asynchronism allows a more robust IC mechanism because of the relative symmetry between users.

By comparing frame synchronous and asynchronous transmission for the simple case of three equal rate users in an isolated cell, we show that user packets do not have to arrive at the base station with the same frame offset to achieve sum rate capacity. We equate the total transmit power for each scheme and assume each user is decoded successfully at the end of their length $T$ frame. We assume the noise level is 1 , and the total received power from all users over the additive white Gaussian noise (AWGN) channel is 7 . Optimum power control is achieved for both schemes when each user has the same signal-tointerference-plus-noise ratio (SINR).

The frame synchronous case is shown on the left of Fig. 1. At the end of the frame (time $t$ ), user 1 is decoded first, followed by user 2 , then user 3. We fix the transmit power of user 3 at 1 , giving it an SINR of 1. If the steady state of optimum power control is attained, each user achieves this SINR with user 2 transmitting at power 2 and user 1 at power 4 . This synchronous SIC scheme achieves a total capacity of $3 \mathrm{~b} / \mathrm{s} / \mathrm{Hz}$ since each user's capacity is $1 \mathrm{~b} / \mathrm{s} / \mathrm{Hz}$ by evaluating $\log _{2}(1+S I N R)$ with an SINR value of 1 . The sum rate for an AWGN channel with a total signal power of 7 and noise power of 1 is 3 $\mathrm{b} / \mathrm{s} / \mathrm{Hz}$ since $\log _{2}(8)$ is 3 . This demonstrates that applying SIC to mutually interfering synchronous CDMA users can achieve sum rate capacity [5].

Now consider the frame asynchronous case on the right of Fig. 1 where the users have evenly distributed timing offsets and the same transmit power. A careful look at the decoding timeline for all three users shows that each user's packet observes the same interference power after canceling the previous (but not future) packets transmitted by the other users. Keeping the total transmit power the same as in the synchronous case requires each user to transmit at power $7 / 3$. At time $t$ we decode user 1 whose frame can be divided into three subframes: (i), (ii), and (iii). Notice that each sub-frame sees a different interference plus noise level of $1,7 / 3+1$, and $2 * 7 / 3$ +1 . If we consider the three subframes as the time-multiplexing of parallel channels with different SINRs, the achievable rate of user 1 (and hence each user by symmetry) can be determined by averaging the capacity of the three parallel channels. The per user capacity is

$$
\begin{aligned}
& \frac{1}{3} \cdot\left[\begin{array}{l}
\left.\log _{2}\left(\frac{7 / 3}{1}\right)+\log _{2}\left(1+\frac{7 / 3}{7 / 3+1}\right)+\right] \\
\log _{2}\left(1+\frac{7 / 3}{2 \cdot 7 / 3+1}\right)
\end{array}\right] \\
& =\frac{1}{3} \log _{2}\left(\frac{10}{3} \cdot \frac{17}{10} \cdot \frac{24}{17}\right)=1,
\end{aligned}
$$

In classic SIC with exponential power shaping and uniform data rates, the last user is received with very low power and is overly dependent on the successful decoding of prior users. Asynchronism allows a more robust IC mechanism because of the relative symmetry between users. 


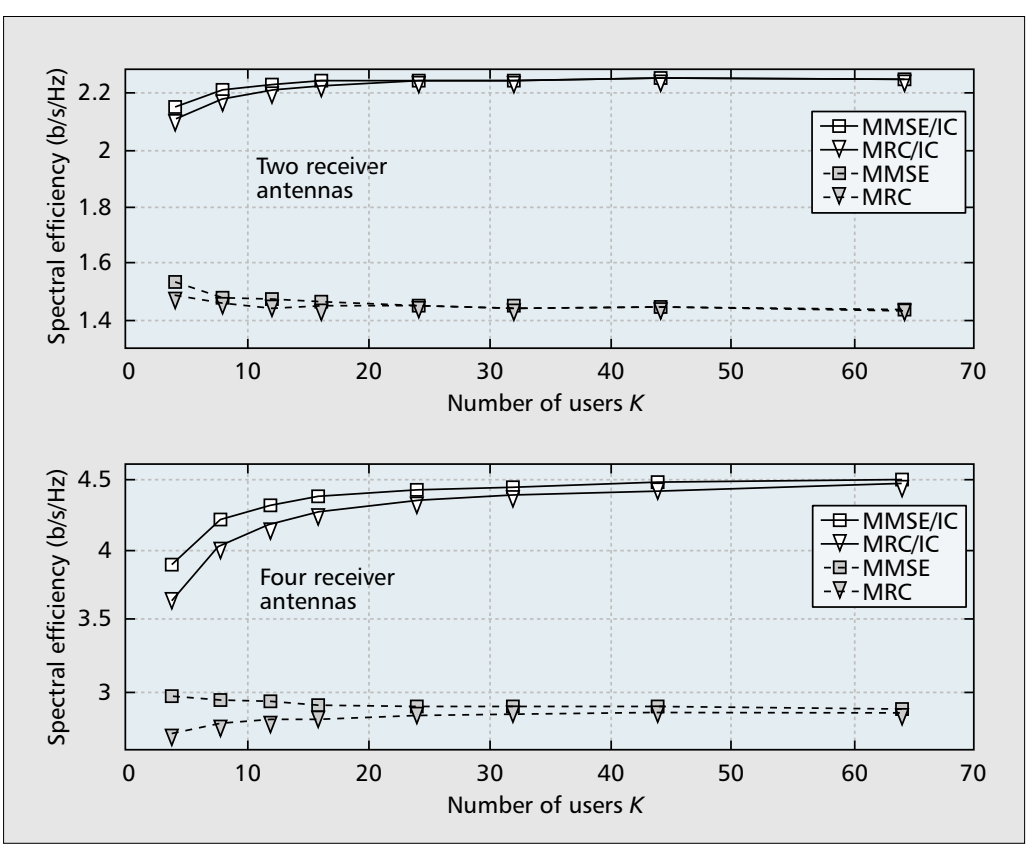

Figure 2. Theoretical CDMA capacity for two (top) and four (bottom) receiver antennas.
(ROT) and is typically controlled to be in the range of 3-10 dB. Operating non-IC CDMA systems at ROT higher than $10 \mathrm{~dB}$ gains little capacity because at that point each user's SINR is governed by interference from other users as opposed to background noise.

In practical systems, ROT is an important parameter for link budget and cell coverage because a new CDMA user entering the system would see a total noise plus interference level that is roughly ROT times greater than the background noise level. Since IC for CDMA improves the SINR of all users, we can operate a CDMA system with IC at an "effective ROT" based on the total power level after cancellation, which better reflects the residual interference and noise level experienced by each user. With effective ROT control users fill up the receiver's cancelled power by transmitting more data power (to get higher data rates) until the level of interference after IC is the same as the level of interference in a non-IC system. This maximizes the capacity while preserving the link budget of all users and hence the network coverage.

\section{Combining the Rich Dimensionality of the CDMA ReVERSE LiNK WITH MULTIPLE BASE \\ StATION RECEIVER ANTENNAS AND INTERFERENCE CANCELLATION}

and it is known [6] that a single coding scheme across these three channels can achieve this rate. The aggregated achievable rate from all three users is 3 , which is equal to the synchronous case and achieves sum capacity. One way to interpret this equivalence is to view the asynchronous case as time-sharing the synchronous case, where the asynchronism deterministically cycles through the three cancellation orderings within each subinterval so that each user spends equal time at three different per user capacities.

Notice from the right of Fig. 1 that the sum rate capacity was achieved by canceling half of the interfering power affecting each user. In the example we removed $1 / 3$ of user 2 's power and $2 / 3$ of user 3 's power to detect user 1 . The interesting frame asynchronous result is that removing half of the interfering power allows the same capacity as if the users were never interfering. For example, if each user took equal turns transmitting at power 7 , each user would get $1 / 3$ of $\log _{2}(1+7 / 1)$ or $1 \mathrm{~b} / \mathrm{s} / \mathrm{Hz}$.

This sum rate achievement of SIC for frame synchronous and asynchronous users can be generalized to an arbitrary number of users, nonuniform offsets, and any power profile, giving a corresponding rate distribution. The result on achieving sum rate also holds for transmissions with variable frame lengths, which occur, for example, in systems with H-ARQ since users can decode after any number of subpacket transmissions.

\section{MAXIMIZING CAPACITY WHILE MAINTAINING COVERAGE WITH EfFective RISE-OVER-TheRMAL CONTROL}

On a classical power controlled CDMA reverse link, the total interference seen by each user is equal to the total received power of all other users plus thermal noise. Normalized to the thermal noise level, the total received user and noise power is referred to as rise-over-thermal
On the CDMA reverse link, each active user on a given carrier transmits over the entire bandwidth, causing its SINR to be inversely proportional to the number of users. With enough users, each user will operate in the linear capacity region (SINR $<<1$ ) where an increase in the user's SINR by some factor causes its supportable data rate to increase by the same factor since $\log _{2}(1+$ SINR $)$ grows linearly with SINR for small SINR.

To improve coverage and capacity for a given number of base stations per unit area, most cellular systems operate with sectorized cells (e.g., 3 sectors/cell) and multiple base station receiver antennas per sector. When the number of receiver antennas is doubled from 2 to 4/sector, the CDMA reverse link capacity will roughly double because each user's SINR approximately doubles [7]. Similarly, when IC of previously decoded packets is implemented at the base station, the SINR of each user increases, causing the sector throughput to roughly grow in proportion to this SINR gain.

These facts are illustrated in Fig. 2 where we plot the results from a simple CDMA capacity analysis with maximal ratio combining (MRC) and minimum mean square error (MMSE) combining with and without SIC, following an analytical approach similar to [8]. The curves are for single path random phase AWGN channels and assume ideal channel coding (i.e., spreading gain efficiency. The analysis includes the effects of other cell interference (with factor $f=0.5$ ) and thermal noise (with an effective ROT of $6 \mathrm{~dB}$ ), but ignores the effects of per user overhead channels and imperfect channel estimation.

As expected, the CDMA reverse link capacity gains from IC (about 55 percent for both 2 and 4 antennas with 20 users) are additive to the capacity gains from doubling antennas. The 20$=1$ ) to convert each user's SINR to spectral 
user spectral efficiency for 4-antenna MMSE/IC is $4.4 \mathrm{~b} / \mathrm{s} / \mathrm{Hz}$, three times larger than the 2-antenna no IC value of $1.45 \mathrm{~b} / \mathrm{s} / \mathrm{Hz}$. After the decoded users have been cancelled from the received samples of each base station antenna with IC, the signal of each remaining user can be MMSE combined across the antennas to achieve combined space-time IC. Indeed, transmitting CDMA and using spatial MMSE processing with SIC achieves sum rate capacity for the case of multiple receiver antennas [9].

With MMSE antenna combining and no SIC, the capacity is largest for a small number of users due to spatial cancellation of interference. With perfect cancellation and no per user overhead, Fig. 2 shows how the CDMA SIC capacity increases with the number of users when the number of receiver antennas is greater than 1 . If the number of receiver antennas is greater than 1 , reverse links that orthogonalize users within a sector (e.g., based on time-division multiple access [TDMA] or orthogonal frequency-division multiplexing [OFDM]) cannot achieve capacity because the receiver (for each time slot or tone) can only achieve a signal-to-noise ratio (SNR) gain from the receiver antennas, not a spatial multiplexing gain. With orthogonal transmission, the degrees of freedom are limited to the single transmit antenna of each user [9]. On the CDMA reverse link, however, when the number of transmitting users is greater than the number of receiver antennas, the dimensionality is not limited by the single transmit antenna of each user. Spatial multiplexing gain is achieved since a CDMA system with $K$ single transmit antenna users is isomorphic to a multiple-input multipleoutput (MIMO) user with $K$ transmit antennas [9]. As technology continually improves to allow better space-time IC, CDMA transmission provides the rich dimensionality at the receiver that is necessary to maximize capacity.

\section{IMPLEMENTING INTERFERENCE CANCELLATION FOR EV-DO REV A WITH ASYNCHRONISM AND HYBRID ARQ: "DON'T FIGHT THE FADING"}

As discussed earlier, sum rate capacity can be achieved through SIC without the need for a specific arrival distribution, power profile, or packet length among users. In practice, IC can be added into base station processing without modification to user terminals or EV-DO standards. On the EV-DO Rev A reverse link, the user frame offsets are intentionally staggered with respect to each other. The base station assigns frame offsets to users in a granularity of one slot with four possible frame offsets.

The time-varying nature of wireless communications channels results in the received power of each user upfading and downfading due to multipath propagation. In fading channels, the capacity is significantly improved by H-ARQ where each packet is broken into a series of smaller subpackets that are transmitted one at a time. Each EV-DO Rev A subpacket is four slots long and there are three interlaces, resulting in eight slots between the end of the subpacket of a given interlace and the beginning of the next subpacket of the same interlace. This gives enough time for the base station to decode the packet and relay an acknowledgment (ACK) or negative ACK (NAK) to the transmitter. The maximum packet length is four subpackets, and each packet can decode correctly after one to four subpackets are received.

Instead of targeting a particular received power profile and IC decoding order among users, it is natural for each user to target a similar packet error rate (PER) but have the fast fading affect which users are more likely to decode at each point in time. This is in fact accomplished by classic CDMA closed loop power control with an inner loop for received pilot SINR and an outer loop that adjusts each user's target pilot SINR to achieve the desired PER on each user's particular channel, thereby compensating for path loss, shadowing, and slow fading. In EV-DO Rev A the transmit pilot of each user is power controlled, while the payload size and latency termination target of each user govern the transmit data power according to the appropriate trafficto-pilot (T2P) ratio [3]. Therefore, IC can be implemented at each CDMA base station without modifying the current operation of reverse link power control which maintains similar received pilot SINR among users.

IC is naturally suited for a CDMA reverse link with $\mathrm{H}$-ARQ. Conventional receivers for $\mathrm{H}-\mathrm{ARQ}$ attempt to decode a given user's packet after each successive subpacket is received. To include IC in this process, the previous subpackets are also redemodulated when a new subpacket is received, with the demodulation occurring on a sample buffer from which decoded packets are being subtracted. Each packet of each user is decoded based on log-likelihood ratios that appropriately soft combine the information from all available subpackets. This is already done in H-ARQ receivers to account for the time-varying fading affecting each subpacket. The additional SINR variance due to IC can easily be taken into account by the decoding process. Combining IC with H-ARQ does not require additional decoding steps relative to conventional H-ARQ receivers, but the results below show that additional decoding can improve performance.

Fast Rayleigh fading does not prevent the efficient implementation of IC because the fading that is faster than closed loop power control simply affects the decoding probabilities of each user after each subpacket. A user who fades up (thereby causing more interference to others) is more likely to decode early and can then be removed before subsequent decoding attempts for remaining users. That is why IC does not have to fight the fast fading, but can instead allow the fading to govern the appropriate order of successful decoding.

\section{RECEIVER ARCHITECTURES FOR INTERFERENCE CANCELLATION}

In this section we describe receiver structures that reconstruct and cancel traffic, pilot, and overhead signals from the base station receiver sample buffer that is shared among users. Since the transmitted pilot sequence is known a priori, the pilot contribution can be reconstructed based on the Rake receiver multipath channel

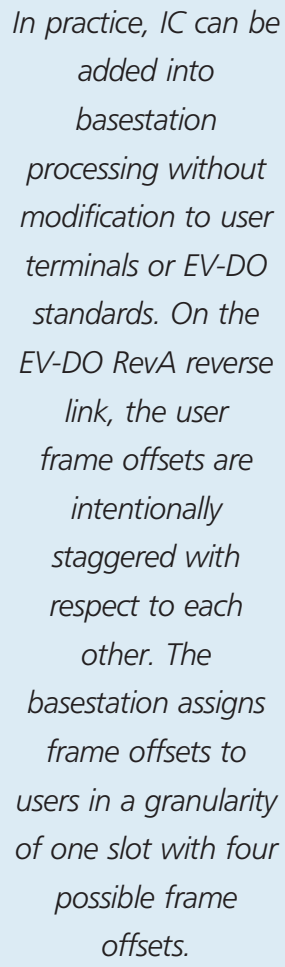




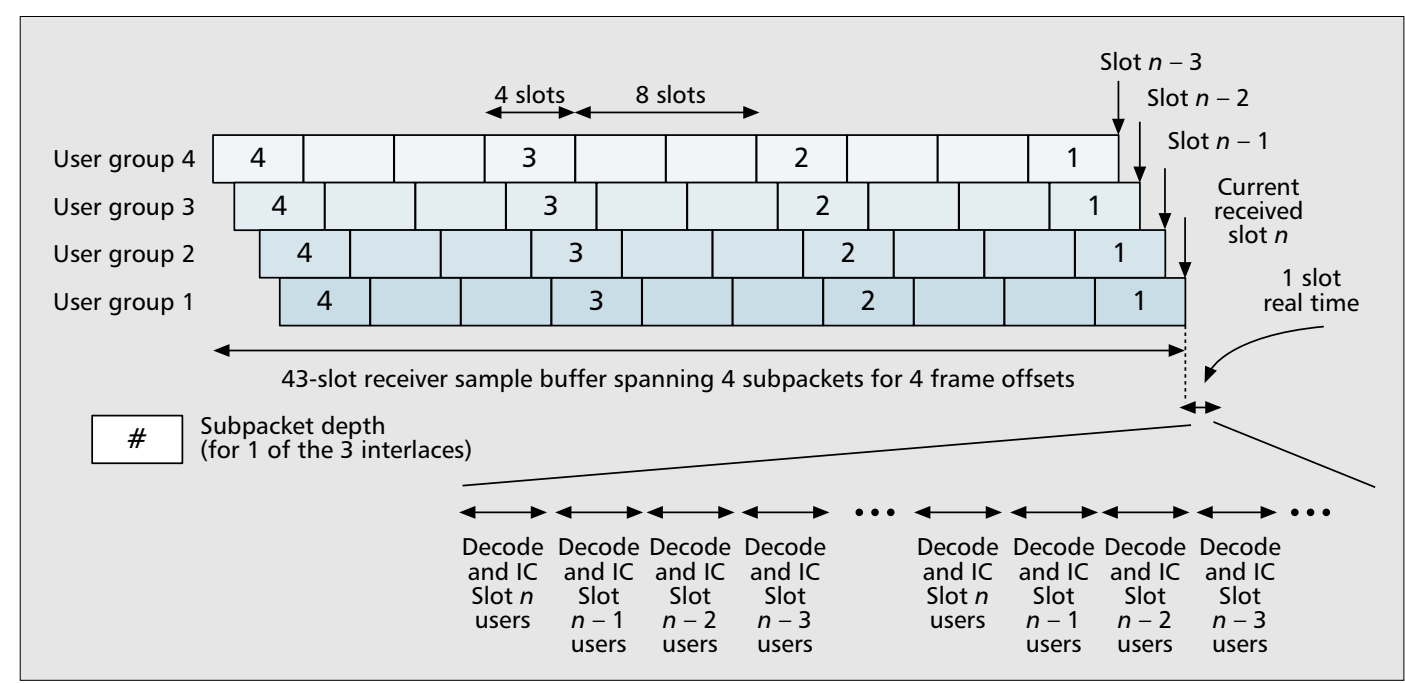

Figure 3. Receiver sample buffer and iterative IC process.

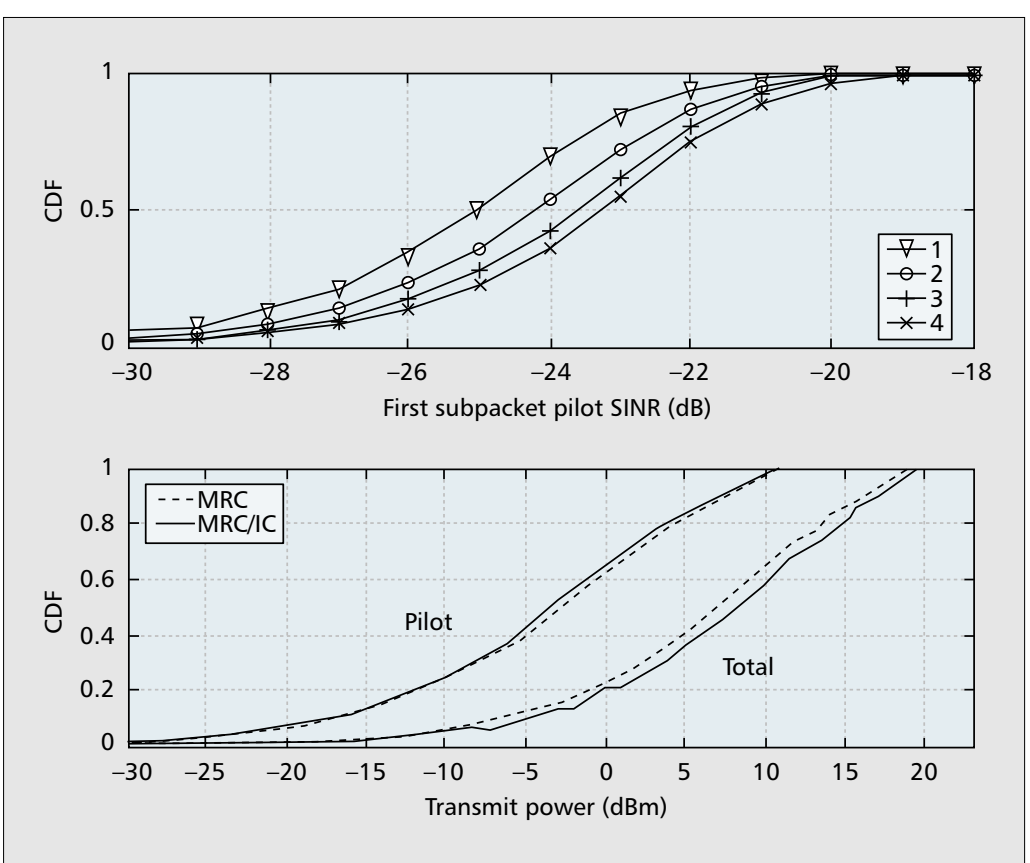

Figure 4. Distribution of 1st subpacket SINR (top) and transmit power (bottom).
$=10 \mathrm{~dB}$ for $38.4 \mathrm{~kb} / \mathrm{s}$ ), the re-encoded traffic symbols can be used to determine improved multipath channel estimates for reconstruction. Our simulation results below use this data-based channel estimation to remove decoded data packets, but remove the pilot, DRC, and RRI channels using the pilot-based channel estimate. The reconstruction of samples in the sample RAM can be determined by convolving the data, pilot, and overhead chip sequences with a combined transmit-receive filter with the timing determined by each Rake finger.

With H-ARQ, each packet is transmitted as a series of at most four subpackets resulting in a 40 -slot time span for one 16 -slot packet. This sets the requirement for the buffer size since the best IC performance is achieved by demodulating each subpacket out of the sample buffer. Similarly, all subpackets of a decoded packet are cancelled from the buffer. Storing 43 slots allows iterative IC that reattempts decoding of users on the previous three frame offsets, as shown in Fig. 3. Attempting to decode packets further than three frame offsets back from the current frame offset could still be useful in terms of removing interference if the packet decodes, but the $\mathrm{H}$ ARQ transmit ACK deadline would be missed. For a base station with 4 antennas/sector and 3 sectors, the amount of RAM memory is 12 antennas $\times 43$ slots $\times 2048$ chips/slot $\times 2$ samples/chip $\times 16 \mathrm{~b} / \mathrm{sample}=32.25 \mathrm{Mb}$, which is very reasonable in embedded DRAM technology.

If the receiver baseband hardware is running at $180 \mathrm{MHz}$, in one slot of real time $(1.66 \mathrm{~ms})$ there are 300,000 clock cycles that can be used to process all the users on the current frame offset as well as iterating back and forth to previous frame offsets. In a practical example we describe a procedure that achieves a good trade-off between performance and complexity by twice iterating over frame offsets $n, n-1, n-2$ as follows:

1. After the current slot (denoted $n$ ) arrives, demodulate and attempt to decode all users whose subpackets finish on that slot.

2. Reconstruct and remove the interference of users who decode successfully in step 1 .

3. Redemodulate and attempt to decode all 
undecoded users whose latest subpackets finish on the previous slot (denoted $n-1$ ).

4. Reconstruct and remove the interference of users who decode successfully in step 3 .

5. Repeat steps 3-4 for users whose latest subpackets finish on slot $n-2$.

6. Repeat steps $1-5$ once.

At the expense of more complexity, further improvements would result from applying SIC among users within a frame offset instead of the group IC described above. In addition, for the SIC approach the decoding order within each frame offset should begin with users who are most likely to decode (e.g., older packets for which more subpackets have been received) and most useful to remove (e.g., high T2P).

\section{Simulation RESULtS}

In this section we provide network level simulation results for the gains from implementing IC on EV-DO Rev A. The simulations are based on the standard evaluation methodology [11], and implement the physical and MAC layers for a network of 19 cells with 3 sectors/cell. Each packet is transmitted with a T2P ratio that targets 1 percent PER after transmission of four subpackets. Results use the standard channel mix [11] where 30 percent of users are in channel A (3 km/hr, 1-path), 30 percent in channel B $(10 \mathrm{~km} / \mathrm{hr}, 3$-path), 20 percent in channel C (30 $\mathrm{km} / \mathrm{hr}, 2$-path), 10 percent in channel D (120 $\mathrm{km} / \mathrm{hr}, 1$-path), and 10 percent in channel E (Rician with $10 \mathrm{~dB} K$-factor).

In terms of regenerating the signals for cancellation, a reconstruction model was incorporated into the simulation to reflect the instantaneous channel estimation impact on IC due to sub-chip multipath fading and Doppler. Data-based channel estimation is used to reconstruct decoded data packets. The pilot, DRC, and RRI channels are cancelled based on pilot estimation. The effective ROT of the system with IC is controlled to be the same as the ROT of the system without IC, both exceeding $7 \mathrm{~dB} 1$ percent of the time. Results are given for an iterative IC receiver described by steps $1-6$ above, a noniterative IC receiver that only implements steps 1 and 2 , and a conventional Rake receiver.

To explain the source of capacity gains Fig. 4 plots the cumulative distribution function (CDF) (over all packets that decode after the fourth subpacket) of the SINR for the first subpacket after the base station has received the first, second, third, and fourth subpacket for noniterative IC with 10 users/sector and 2 antennas/sector. As the subpacket ages and moves through the buffer, its SINR improves since other users are decoded and cancelled during the time period between successive subpacket arrivals. With $\mathrm{H}$ ARQ and IC, not only does the receiver get the extra energy from the newest subpacket, but the SINR of the older subpackets gets better. By the time the last subpacket is received, the median SINR of the first subpacket has improved by almost $2 \mathrm{~dB}$. Figure 4 also includes the CDF of transmitted pilot power and total (i.e., pilot, data, and overhead) power for the noniterative IC and Rake receivers. Adding IC does not result in increased transmitted pilot power of

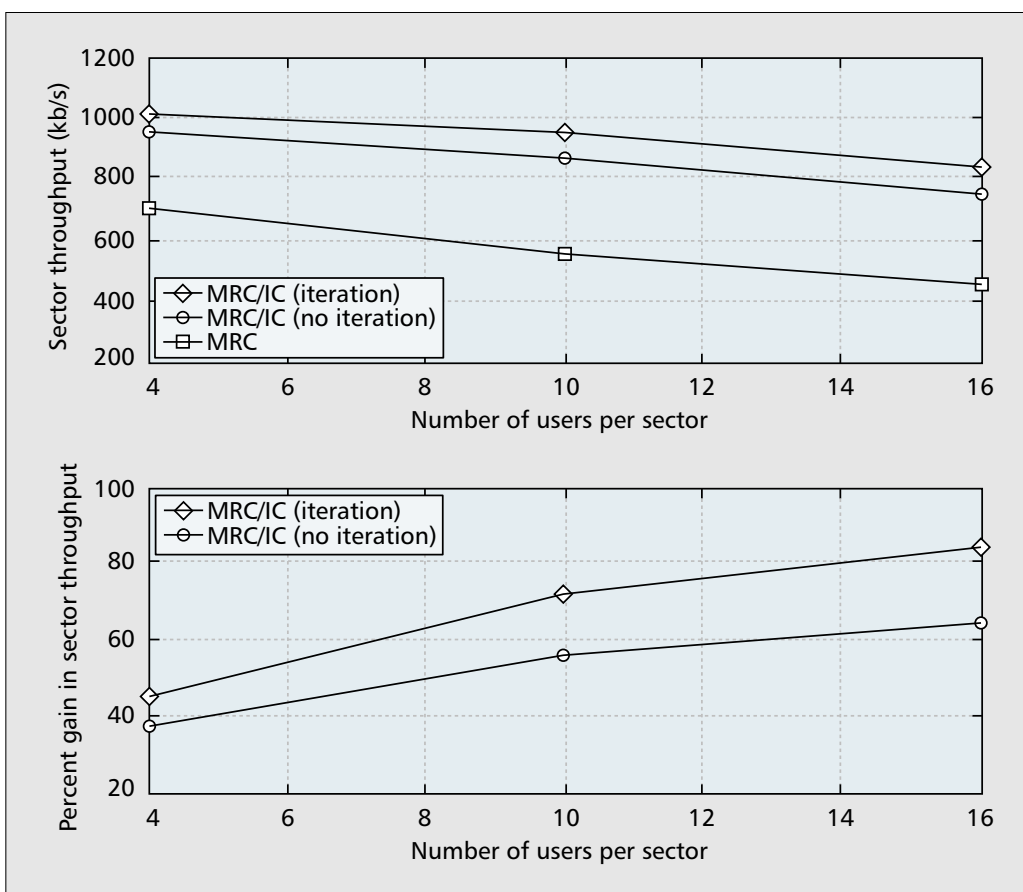

Figure 5. Sector throughput (top) and gains from IC (bottom).

users. This means that a link budget limited user at the cell edge can maintain the same data rate by transmitting at its maximum power (e.g., 23 $\mathrm{dBm}$ ) in both the non-IC and IC cases, proving that network coverage has been maintained by effective ROT control. The total transmit power is higher for IC because users are transmitting more data power (higher T2P) to achieve higher data rates.

In Fig. 5 we plot the sector throughput (top) and corresponding IC capacity gain (bottom) for iterative and noniterative IC with two receiver antennas. The data throughput values decrease as more users are added since each user transmits pilot and overhead channels that do not count toward throughput. The gains of IC over the conventional receiver increase with the number of users since canceling pilot, overhead, and data all allow more data to be transmitted. Compared to processing only the current frame offset users, iterating between the current and previous two frame offsets allowed additional users to decode and be cancelled. With 16 users/sector, iterating IC increased the capacity gain from 65 to 84 percent.

Figure 6 considers iterative IC for two and four receiver antennas with 10 users/sector. The effective ROT distribution plotted for the IC receiver is seen to be very similar to the ROT distribution plotted for the non-IC receiver. This demonstrates that IC does not affect system stability. The per user throughput distributions show the data rate gain from IC being realized by users at all data rates. IC results are given with and without perfect reconstruction, showing that the system effect of imperfect cancellation amounts to about 15 percent in sector throughput. The gains from IC are significant for both two and four receiver antennas. Doubling the number of antennas allowed the IC throughput to increase from 956 to $1999 \mathrm{~kb} / \mathrm{s}$. Relative to 


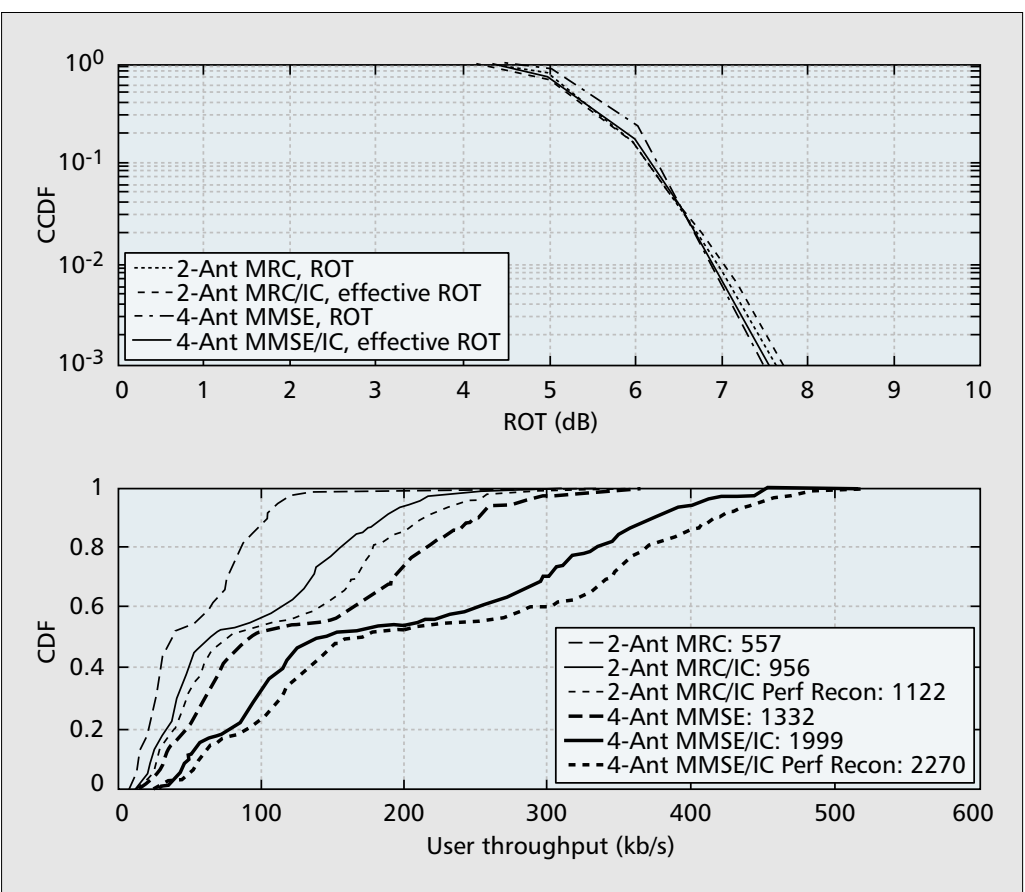

Figure 6. Distribution of ROT and effective ROT (top) and user throughput (bottom).

\section{REFERENCES}

[1] "cdma2000 High Rate Packet Data Air Interface Specification - Revision A," 3GPP2 C.S20024-A, v. 1.0, Mar. 2004.

[2] N. Bhushan et al., "cdma2000 1xEV-DO Revision A: A Physical and MAC Layer Overview," IEEE Commun. Mag., this issue.

[3] M. Fan et al., "On the Reverse Link Performance of cdma2000 1xEV-DO Revision A System," Proc. IEEE ICC 2005, vol. 4, pp. 2244-50.

[4] S. Verdu, Multiuser Detection, Cambridge Univ. Press, 1998.

[5] A. J. Viterbi, "Very Low Rate Convolutional Codes for Maximum Theoretical Performance of Spread-Spectrum Multiple-Access Channels," IEEE JSAC, vol. 8, no.4, May 1990, pp. 641-49.

[6] R. G. Gallager, Information Theory and Reliable Communications, Wiley, 1968.

[7] Y. Tokgoz, M. Fan, and J. Smee, "Reverse Link Performance of cdma2000 1xEV-DO Revision A System with Antenna Array Receivers," IEEE GLOBECOM 2005.

[8] S. Verdu and S. Shamai (Shitz), "Spectral Efficiency of CDMA with Random Spreading," IEEE Trans. Info. Theory, vol. 45, no. 2, Mar. 1999, pp. 622-40.

[9] D. Tse and P. Viswanath, Fundamentals of Wireless Communications, Cambridge Univ. Press, 2005

[10] M. Yavuz et al., "VolP over cdma2000 1xEV-DO Revision A," IEEE Commun. Mag., this issue.

[11] 3GPP2 TSG-C WG3, "1xEV-DO Evaluation Methodology," 3GPP2 TSG-C C30-20041002-004, Oct. 2004.

\section{BIOGRAPHIES}

JILEI Hou [M] (jhou@qualcomm.com) received his B.E. degree (with Honor) and M.E. degree in electrical engineering from Southeast University, Nanjing, China, in 1995 and 1998, respectively, and a Ph.D. degree in electrical engineering from the University of California at San Diego, La Jolla, in 2003. He joined the Corporate Research and Development Division of QUALCOMM Incorporated in May 2003 where he has been actively involved in the performance analysis, system design, and development of advanced receivers for high data rate mobile wireless systems. His research interests include communication theory, information theory, channel coding, spread spectrum, and spacetime communications.

IC can readily be applied to commercial EV-DO Rev A systems to give capacity gains without modifications to user terminals or standards. We have illustrated how the classical information theory results on achieving sum rate capacity with CDMA and SIC can be translated into designing practical receivers that support multiple antennas, asynchronism, and $\mathrm{H}-\mathrm{ARQ}$, and offer significant capacity gains on multipath fading channels. With each CDMA user in the linear capacity region, SINR gains from space-time IC translate directly into capacity gain, allowing the system to support more users at higher data rates and/or lower latencies. As VLSI and DSP processing speeds continue to scale to allow better space-time IC, it is clear that a CDMA transmission scheme provides the rich dimensionality at the receiver necessary to achieve high spectral efficiency. Compared to today's base stations that have two-way receive diversity without IC, by moving to four-way receive diversity and IC, reverse link capacity can be increased by a factor greater than 3 . Such gains do not require standards changes, are fully attainable with existing EV-DO Rev A terminals, and can be selectively deployed by an operator depending on capacity requirements.

\section{ACKNOWLEDGMENTS}

The authors thank Peter Black, Roberto Padovani, and anonymous reviewers for their constructive comments that significantly improved this article, and also thank Joseph Soriaga for providing simulation results.
JoHn E. SMEE [SM] (jsmee@qualcomm.com) is a senior staff engineer and manager in the Corporate Research and Development group at QUALCOMM, Inc. He received his B.Sc. and M.Sc. from Queen's University, Kingston, Canada, in 1993 and 1995, and his M.A. and Ph.D. from Princeton University, New Jersey, in 1997 and 2000, all in electrical engineering. He joined QUALCOMM in January 2000, first working on the design and development of adaptive space-time equalization for EV-DO mobile station modems. He has been involved in system design for a variety of projects focused on innovation of wireless communications systems, making contributions to CDMA, 802.11, OFDM, and MIMO. He is currently leading the systems design for CDMA interference cancellation. He has been granted seven patents in the area of digital communications.

HenRY D. Pfister received a B.S. degree in physics in 1995 an M.S. degree in electrical engineering in 2000, and a Ph.D. degree in electrical engineering in 2003, all from the University of California at San Diego. From 2003 to2004 he worked as a senior engineer for QUALCOMM, Inc. San Diego, California. Since January 2005 he has been a scientific collaborator at the Swiss Federal Institute of Technology, Lausanne.

StefANO TOMAsIn [M'00] (tomasin@dei.unipd.it) is an assis tant professor at the University of Padova, Italy, where he received Laurea (1999) and Ph.D. (2002) degrees in telecommunications engineering. In 1999 he was a visiting student at the IBM Research Laboratory, Zurich, Switzerland, working on signal processing for magnetic recording systems. In academic year 2001-2002 he was with Philips Research, Eindhoven, the Netherlands, studying multicarrier transmission for mobile applications. In 2002 he joined the University of Padova, first as a contractor researcher for a national research project and then as an assistant professor. In the second half of 2004 he was visiting faculty at QUALCOMM, San Diego, working on interference cancellation for CDMA systems. 